Research, Society and Development, v. 9, n. 7, e939975183, 2020

(CC BY 4.0) | ISSN 2525-3409 | DOI: http://dx.doi.org/10.33448/rsd-v9i7.5183

\title{
Uso do paclobutrazol na produção de manga
}

Use of paclobutrazol in mango production

\section{Uso de paclobutrazol en la producción de mango}

Recebido: 02/06/2020 | Revisado: 02/06/2020 | Aceito: 04/06/2020 | Publicado: 16/06/2020

\section{Girlaine Pereira Oliveira}

ORCID: https://orcid.org/0000-0003-3104-0112

Universidade Federal do Espírito Santo, Brasil

E-mail: layneagro@gmail.com

\section{Resumo}

O uso de reguladores de crescimento é essencial para o sucesso da mangicultura no Brasil, uma vez que desempenham importante função na indução floral, permitindo o escalonamento da produção durante o ano todo e obtenção de frutos de boa qualidade e quantidades que atendam a demanda interna e externa. Devido à grande importância econômica da manga essa revisão objetiva esclarecer aspectos sobre o uso de paclobutrazol na indução do florescimento da mangueira. A metodologia adotada foi o estudo bibliográfico, sendo as informações obtidas em fontes documentais. Verificou-se que a temperatura é o fator climático mais importante para o florescimento da mangueira porque as temperaturas baixas paralisam o crescimento vegetativo que é indispensável para o florescimento. As giberelinas (GAs) são hormônios vegetais que regulam o florescimento, em altas concentrações favorecem o crescimento vegetativo e em baixas concentrações no interior dos ramos induzem o florescimento. Por isso, os produtos que interferem na síntese de GAs se mostram eficientes na indução do floração e dentre os produtos inibidores da síntese de GAs, o paclobutrazol (PBZ) é o mais utilizado na produção de manga. O PBZ é aplicado no solo úmido, após dois surtos de brotação das plantas, por isso, a poda após colheita é essencial para que essas brotações sejam vigorosas e uniformes. Para muitas regiões tradicionalmente produtoras de manga as doses de PBZ já foram definidas e dependem da cultivar, tipo de solo, condições de cultivo, além da condição nutricional das plantas e variam de 0,3 a 1,62 gramas por metro linear da copa das plantas. Para o sucesso da indução floral da mangueira a aplicação de PBZ deve ser aliado a outros manejos como poda, nutrição mineral e irrigação.

Palavras-chave: PBZ; Florescimento; Fenologia; Mangueira. 


\begin{abstract}
The use of growth regulators is essential for the success of mangiculture in Brazil, since they play an important role in floral induction, allowing the staggering of production throughout the year and obtaining good quality fruits and quantities that meet the internal demand and external. Due to the great economic importance of the mango, this review aims to clarify aspects about the use of paclobutrazol in inducing the flowering of the mango. The methodology adopted was the bibliographic study, and the information was obtained from documentary sources. It was found that temperature is the most important climatic factor for the flowering of the mango tree because low temperatures paralyze the vegetative growth that is indispensable for flowering. Gibberellins (GAs) are plant hormones that regulate flowering, in high concentrations favor vegetative growth and in low concentrations inside the branches induce flowering. Therefore, products that interfere in the synthesis of GAs are efficient in inducing flowering and among the products that inhibit GAs, paclobutrazol (PBZ) is the most used in mango production. It is applied to moist soil after two outbreaks of plant sprouting, therefore pruning after harvest is essential for these shoots to be vigorous and uniform. For many traditionally mango producing regions, the doses of PBZ have already been defined and depend on the cultivar, soil type, cultivation conditions, in addition to the nutritional condition of the plants and vary from 0.3 to 1.62 grams per linear meter of the canopy. For the success of floral induction of the mango tree, the application of PBZ must be combined with other managements such as pruning, mineral nutrition and irrigation.
\end{abstract}

Keywords: PBZ; Flowering; Phenology; Mango tree.

\title{
Resumen
}

El uso de reguladores del crecimiento es esencial para el éxito de la manicultura en Brasil, ya que juegan un papel importante en la inducción floral, permitiendo el escalonamiento de la producción durante todo el año y la obtención de frutas y cantidades de buena calidad que satisfacen la demanda interna y externo. Debido la gran importancia económica del mango, la revisión tiene como objetivo aclarar aspectos sobre el uso de paclobutrazol para inducir la floración del mango. La metodología adoptada fue el estudio bibliográfico, y la información se obtuvo de fuentes documentales. Se descubrió que la temperatura es el factor climático más importante para la floración del árbol de mango porque las bajas temperaturas paralizan el crecimiento vegetativo que es indispensable para la floración. Las giberelinas (GAs) son hormonas vegetales que regulan la floración, en altas concentraciones favorecen el crecimiento vegetativo y en bajas concentraciones dentro de las ramas inducen la floración. Por lo tanto, los productos que interfieren en la síntesis de GAs son eficientes para inducir la floración y entre los productos que inhiben los GAs. Paclobutrazol (PBZ) es el más utilizado en la producción de mango y se aplica al suelo húmedo después de dos brotes de brotación de plantas, por lo tanto, la poda después de la cosecha es esencial para que estos brotes sean vigorosos y 
uniformes. Para muchas regiones tradicionalmente productoras de mango, las dosis de PBZ ya se han definido y dependen del cultivar, el tipo de suelo, las condiciones de cultivo, además de la condición nutricional de las plantas y varían de 0.3 a 1.62 gramos por metro lineal del dosel plantas. Para el éxito de la inducción floral de la manguera, la aplicación de PBZ debe combinarse con otras gestiones como la poda, la nutrición mineral y el riego.

Palabras clave: PBZ; Floración; Fenología; Mango.

\section{Introdução}

A área cultivada com mangueiras no Brasil foi de 76,661 mil hectares, gerando uma produção superior a 605.956 toneladas, sendo as regiões Nordeste e Sudeste responsáveis por $66,5 \%$ e $28,4 \%$ da produção total, respectivamente. A exportação de manga bateram recorde em 2019, até o mês de novembro sendo exportados 197 mil toneladas, e os principais destinos a União Europeia e para os Estados Unidos (Anuário, 2019).

O uso de reguladores de crescimento é essencial para o sucesso da mangicultura no Brasil, uma vez que desempenham importante função na indução floral, permitindo o escalonamento da produção durante o ano todo e obtenção de frutos de boa qualidade e quantidades que atendam a demanda interna e externa. Nas regiões semiáridas, principalmente com as cultivares de consumo in natura como a Tommy Atkins e Palmer, para as quais foram definidas doses, época e forma de aplicação, como parte das tecnologias visando à indução floral (Albuquerque e Mouco, 2005; Mouco et al., 2010; Coelho et al.,2014; Oliveira et al., 2015) e em cultivar destinada a indústria de suco também foi realizado com o intuito de uniformizar a floração e a colheita (Oliveira et al., 2017). Em 2019 o escalonamento da produção de manga foi eficaz na região mineira, evitando a concentração de colheita e, consequentemente, queda nos preços (Anuário, 2019).

O paclobutrazol (PBZ) é um regulador de crescimento vegetal que atua reduzindo a biossíntese de giberelinas (Taiz \& Zeiger, 2017), contribuindo para inibição do crescimento dos ramos, promovendo a maturação das gemas, e consequentemente favorecendo a floração (Davenport \& Nuñez-Elizea, 1997).

Devido à grande importância econômica da manga tanto para consumo ao natural quanto para a agroindústria de sucos no Brasil, essa revisão objetiva esclarecer aspectos sobre a fenologia da mangueira e o uso de paclobutrazol na indução do florescimento. 


\section{Metodologia}

O presente trabalho é descritivo, sendo realizada uma revisão da literatura acerca do uso de paclobutrazol (PBZ) na indução do florescimento da mangueira caracterizando os afeitos do produto na fenologia e respostas fisiológicas da planta.

A metodologia adotada foi o estudo bibliográfico sendo as informações obtidas em documentos científicos, principalmente artigos científicos nacionais e internacionais, dissertações e teses. $\mathrm{O}$ trabalho apresenta natureza qualitativa caracterizado pela interpretação por parte do autor com suas opiniões sobre o fenômeno em estudo (Pereira et al., 2018). No primeiro momento foi realizado a coleta de dados, que consistiu da determinação e a separação do material científico referente ao uso do paclobutrazol na produção da mangueira. Terminando a coleta de informações sobre o tema, deu-se a organização do estudo, sendo estabelecido a ligação entre os fatos descrito por diversos pesquisadores e a importância da aplicação do paclobutrazol na produção de manga no Brasil. Após a seleção dos documentos científicos foram seguidos os seguintes passos: leitura exploratória, leitura seletiva, leitura analítica, leitura interpretativa e redação.

\section{Fenologia da Mangueira}

\subsection{Crescimento e desenvolvimento}

A mangueira é considerada uma espécie predominantemente tropical, embora seja capaz de crescer e reproduzir em áreas de latitudes subtropicais com estações secas definidas e alta acumulação térmica. De maneira geral, a mangueira adapta-se e produz bem em ambientes com temperaturas amenas $\left(25^{\circ} \mathrm{C}\right.$ diurno e $15{ }^{\circ} \mathrm{C}$ noturno $)$ e período seco antes da florada (Pinto et al., 2009). Segundo Ramírez e Davenport (2010a) temperatura de $-6{ }^{\circ} \mathrm{C}$ causa morte de mangueiras e acima de $40^{\circ} \mathrm{C}$ causa estresse térmico.

O crescimento da mangueira não é contínuo, ocorre em surtos, e a mangueira só lança um novo surto após a maturação do surto de crescimento anterior (Pinto et al. 2009). A temperatura é o fator climático mais importante para o florescimento da mangueira porque as temperaturas baixas paralisam o crescimento vegetativo que é indispensável para o florescimento (Pinto et al., 2004). A combinação adequada de temperaturas diurnas e noturnas para a floração da mangueira é função da sua adaptação às condições ambientais de cada região, em regiões tropicais a combinação de temperaturas diurnas/noturnas de $30 / 25{ }^{\circ} \mathrm{C}$ estimulam crescimento vegetativo, enquanto a combinação $28 / 18^{\circ} \mathrm{C}$ estimula a intensa floração (Davenport, 2007). A frutificação e o vingamento de frutos também são 
influenciados pela temperatura, sendo as temperaturas abaixo de $16{ }^{\circ} \mathrm{C}$ afetam a produção e a viabilidade do pólen, causando um baixo vingamento de frutos (Pinto et al, 2004).

Fatores como sistema radicular profundo, raízes superficiais resistentes ao dessecamento e sistemas de canais de látex, conferem a mangueira capacidade de sobreviver em ambientes de deficiência hídrica e alta demanda evapotraspiratória por períodos prolongados (Whiley e Schaffer, 1997), por isso, a mangueira é considerada uma planta resistente à seca. No entanto, em plantios comerciais, o cultivo da mangueira demanda precipitação anual mínima de $700 \mathrm{~mm}$ com uma estação seca para promover a indução floral (Mouco et al., 2010).

Nos trópicos de baixa latitude, onde não têm épocas de baixas temperaturas, o estresse hídrico tem sido considerado como um fator de indução do florescimento da mangueira. $\mathrm{O}$ estresse hídrico em mangueiras evita a emissão de fluxos vegetativos favorecendo o amadurecimento dos ramos e portanto, aumentando o tempo para acumulação do estímulo floral. O início do desenvolvimento de gemas reprodutivas ocorrem após um período de 6 a 12 semanas de déficit hídrico (Davenport e Núnez-Elisea, 1997).

Chuvas intensas causam prejuízo ao florescimento da mangueira devido ao estímulo a um vigoroso crescimento vegetativo (Davenport, 2006) e aumentam a proliferação de doenças como antracnose e oídio.

\subsection{Iniciação floral e florescimento}

É importante saber que mangueiras produzem três tipos de brotações: ramos vegetativos, que possuem apenas folhas; reprodutivos, que possuem apenas flores nas inflorescências; e ramos mistos que produzem tanto folhas quanto flores na mesma panícula (Figura 1) (Davenport, 2006; Ramírez e Davenport, 2010). 

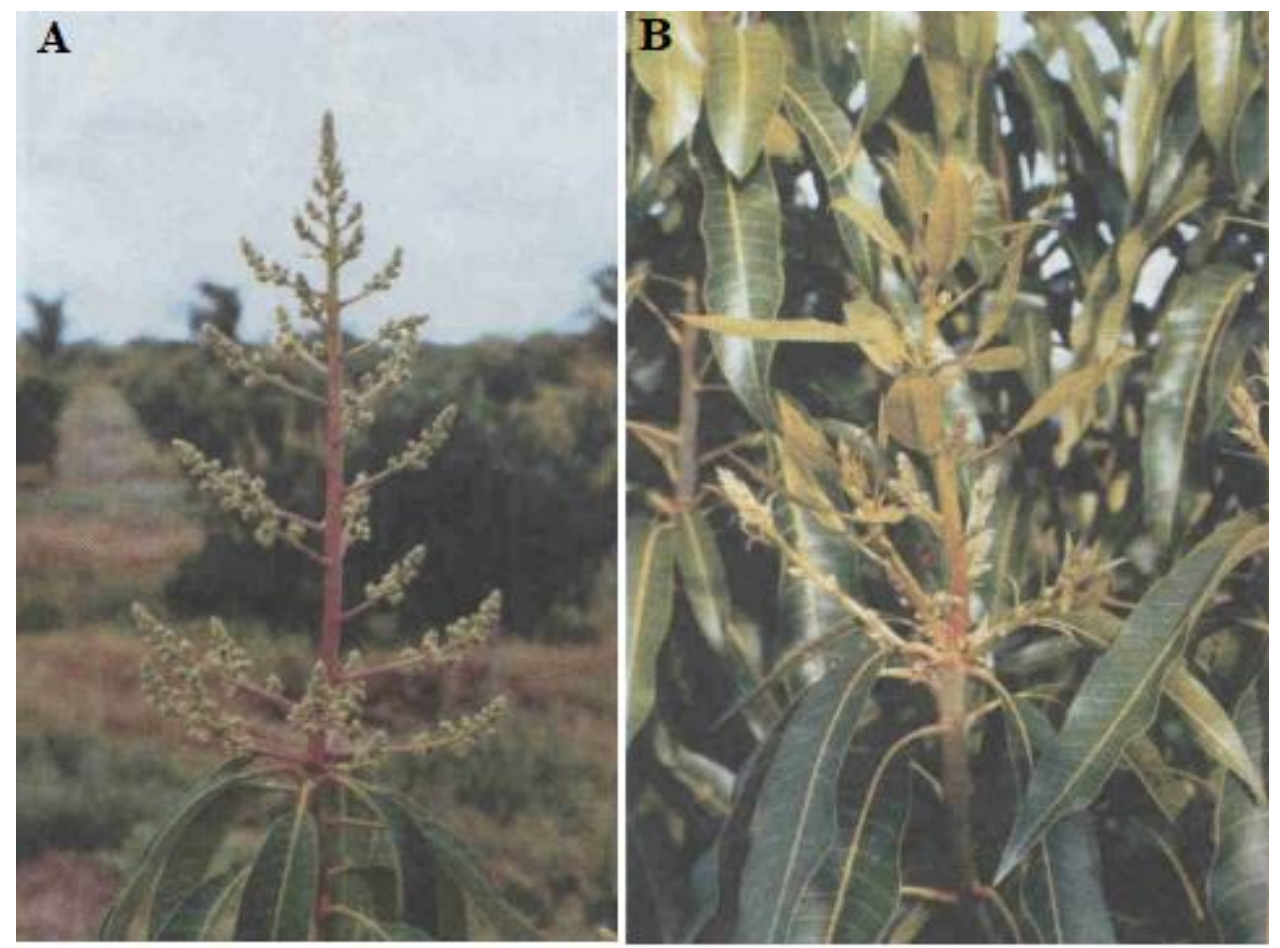

Figura 1. Ramo com panícula normal (A) e ramos com panícula mista (B). Foto: Albuquerque e Mouco (2000).

A iniciação floral em mangueira pode ser induzida por fatores ambientais, como mudança de temperatura e pode ser estimulada por poda, manejo de irrigação, aplicação de reguladores de crescimento e exposição ao etileno (Ramírez e Davenport, 2010). Estudos com indução de florescimento em mangueiras elucidaram a teoria da existência de uma substância denominada "promotor florigênico" (PF) e outra substância denominada "promotor vegetativo" (PV) que se acredita ser uma giberelina. O suposto PV estimula a emissão de fluxos vegetativos e o PF estimula a emissão de fluxos reprodutivos (Ramírez e Davenport, 2010). O promotor florigênico (PF) da mangueira é sintetizado nas folhas e regulado pela temperatura. Em condições de baixas temperaturas, nas regiões subtropicais ocorre o aumento do promotor florigênico (PF) e a diminuição do promotor vegetativo (PV), que resulta no aumento da razão PF/PV que resulta na indução floral (Ramírez e Davenport, 2010). Elevada razão PF/PV estimula a formação de brotações reprodutivas, enquanto que a baixa relação PF/PV estimula a formação de brotações vegetativas e, em níveis intermediários brotações mistas são induzidas (Ramírez e Davenport, 2010; Ramírez et al, 2010a). O PF é translocado para as gemas via floema, pois ramos anelados e completamente desfolhados não florescem (Ramírez e Davenport, 2010; Ramírez et al, 2010a).

As giberelinas são hormônios vegetais que parecem ser os promotores vegetativos e atuam na regulação da floração da mangueira. Altos níveis de giberelinas inibem a floração e 
estimulam o crescimento vegetativo, enquanto o declínio nos teores de giberelinas aumenta a floração (Ramírez et al, 2010a). Os reguladores de crescimento vegetal que interferem na síntese de giberelinas (GAs) são: os compostos quaternários, como o cloreto de mepiquat e o cloreto de chlormequat (CCC), os compostos cíclicos contendo nitrogênio, como o paclobutrazol (PBZ) e o uniconazole, e os acilciclohexanodionas, como o etiltrinexapac (TrixE) e o prohexadione-Ca (ProCa), que podem bloquear as reações finais da síntese de giberelinas (Asin et al., 2007).

O florescimento pode ocorrer em regiões tropicais após um período de estresse hídrico, no entanto, o estresse hídrico não induz a floração. O estresse hídrico mantém a árvore em repouso e impede a iniciação de um novo fluxo de crescimento, diminuindo os níveis de giberelinas $(\mathrm{PV})$ e, consequentemente ocorre à maturação dos ramos (Ramírez e Davenport, 2010), permitindo o acúmulo do promotor florigênico à medida que o ramo amadurece (Silva, 2014). Em razão disso, a maturação do último surto de crescimento impactado pela duração do estresse hídrico é que induz o florescimento.

Em regiões de climas tropicais, a idade do último fluxo de ramos é o principal fator que determina a indução floral. À medida que aumenta a maturidade dos ramos o florescimento torna-se mais provável e, ocorre em ramos terminais que apresentam coloração verde escuro, indicativo que alcançou idade mínima de quatro meses (Ramírez e Davenport, 2010; Ramírez et al, 2010b).

De acordo com Santos-Villalobos et al., (2013), o florescimento em mangueiras pode ser regulado por fatores hormonais e por carboidratos. Fitohormônios como as auxinas, citocininas e giberelinas parecem regular a sincronização do desenvolvimento de gemas, bem como a determinação da direção de seu desenvolvimento como gema floral ou vegetativa. Sendo assim, a indução do florescimento pode ser resultante do acúmulo de níveis ótimos de carboidratos nas gemas, os quais, associados a supostos estímulos florais, desencadeariam a indução floral (Davenport, 2007). Estudos desenvolvido com a mangueira 'Ubá' e 'Ataulfo', (Oliveira et al., 2018; Santos-Villalobos et al., 2013) observaram redução nos níveis de carboidratos nas folhas ao longo do tempo, em determinados períodos do ano, caracterizados pela ocorrência de floração. Os autores associaram a redução nos níveis de carboidratos nas folhas ao aumento do desenvolvimento das gemas florais, o que não ocorria em outros períodos, em que nenhum ou pouco florescimento era observado. Estes resultados sugerem que o conteúdo de carboidratos nas folhas está, de fato, envolvido no desenvolvimento das gemas florais em mangueira em função da demanda energética para formação das inflorescências. 


\section{Uso do paclobutrazol na mangueira}

O paclobutrazol é uma substância do grupo químico dos triazóis, com classificação toxicológica classe III (Costa et al., 2008) (Figura 2). Ele atua reduzindo a síntese de giberelinas, pois interfere nos três primeiros passos da síntese, inibindo a oxidação caureno e impedindo a formação de ent-caurenol, entcaurenal e ácido ent-caurenoico. Essas reações são catalisadas pela enzima caureno oxidase, que é inibida pela ação dos triazóis (Taiz e Zeinger, 2017). O bloqueio da síntese de giberelinas resulta na redução do crescimento vegetativo da mangueira promovendo o florescimento.

O uso do paclobutrazol em mangueiras é bastante difundido nas regiões tropicais do mundo e consiste na aplicação do produto via solo, após a poda, possibilitando o escalonamento de produção ao longo do ano (Upreti et al., 2013), porém, a aplicação via foliar do PBZ é ineficiente em regular o crescimento vegetativo e promover o florescimento da mangueira (Alburquerque e Mouco, 2005). Em trabalhos realizados com mangueiras foi verificado que a aplicação do PBZ nos solo, reduziu o crescimento dos ramos, reforçando as informações sobre a efetividade do produto em reduzir ou regular o crescimento vegetativo (Oliveira et al., 2015; Garcia de Niz et al., 2014).

Vale ressaltar que o manejo do crescimento vegetativo é de grande importância na produção de fruteiras, já que, evitando a brotação excessiva, é possível promover a floração e a frutificação precoce em plantas jovens (Davenport, 2007), além de melhorar a eficiência do controle de pragas e doenças na copa das árvores.

A aplicação do paclobutrazol deve ser realizada no solo úmido, já que a água é o veículo de condução do produto. O produto deve ser diluído em um a dois litros de água por planta, para facilitar a distribuição ao redor da planta (Albuquerque et al, 1999). A locomoção lenta do paclobutrazol dentro da planta sugere que o mesmo deve ser aplicado após a emissão do segundo fluxo de crescimento depois da poda de ponteiros (Albuquerque et al., 2002), com os ramos ainda imaturos (Figura 2). 


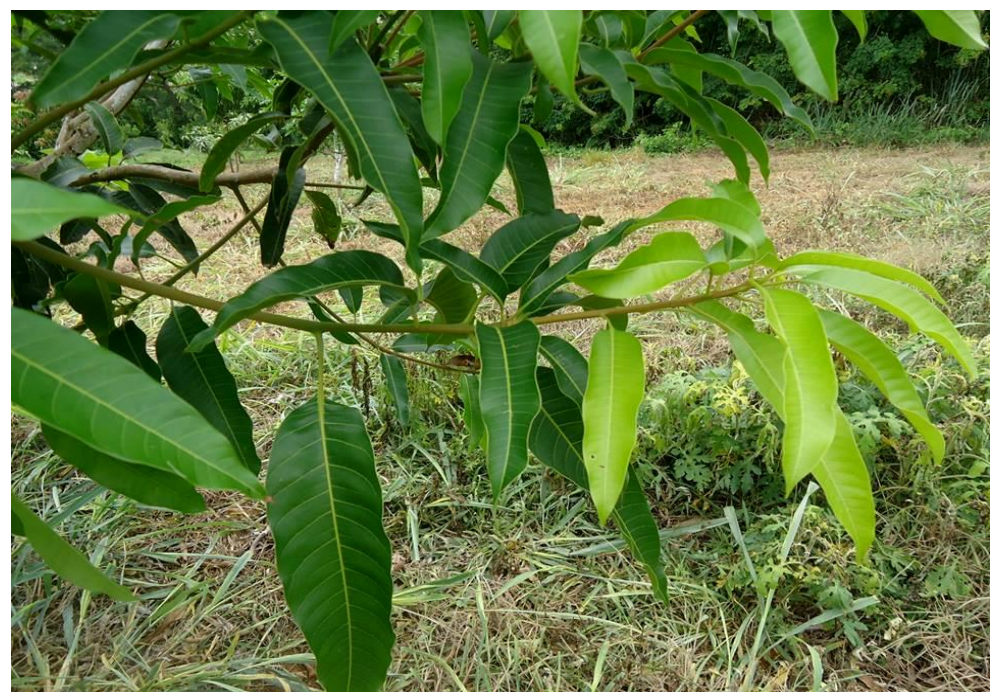

Figura 2. Fluxos vegetativos de mangueiras 'Ubá' no momento da aplicação do paclobutrazol. Foto: Oliveira (2015).

Respostas diferentes a aplicação de inibidores de giberelinas podem ser obtidas na cultura da mangueira. A dosagem de paclobutrazol pode variar, dependendo da cultivar. Cultivares que possuem intensa capacidade de vegetar necessitam de maiores doses (Albuquerque et al., 2002; Oliveira et al., 2017), como é o caso das cultivares Keitt, Kent e Ubá, e dentro da mesma variedade a sensibilidade vai depender da época de aplicação, idade e nutrição das plantas. Em geral, plantas mais jovens são mais sensíveis e respondem a menores doses de paclobutrazol do que as plantas mais velhas (Albuquerque e Mouco, 2000). Por esses motivos, é necessário estudos para cada cultivar e região para obter melhor retorno econômico com a prática de indução floral (Quadro 1).

Na prática, a copa da árvore é medida de uma extremidade a outra e a dosagem é definida em gramas do ingrediente ativo por metro linear da copa. 
Quadro 1. Dose de paclobutrazol em gramas do ingrediente ativo por metro linear da copa para as variedades de mangueira

\begin{tabular}{|l|l|l|}
\hline Variedade & $\begin{array}{l}\text { g de PBZ/metro linear da } \\
\text { copa }\end{array}$ & Referência \\
\hline Tommy Atkins & 1,50 & Coelho et al. (2014) \\
\hline Palmer & 0,30 & Oliveira et al. (2015) \\
\hline Keitt & 1,30 & Simões et al. (2017) \\
\hline Ubá & 1,62 & Oliveira et al. (2017) \\
\hline Bourbon & 0,80 & Chatzivagiannis et et al. \\
& & Husen et al. (2012) \\
\hline Híbridos de Arumanis - & 1,25 & Cardoso et al. (2007) \\
\hline 143 & 0,80 & \\
\hline Rosa & & \\
\hline
\end{tabular}

Segundo Mouco e Alburquerque (2005) o uso de paclobutrazol só é eficiente nas épocas quentes e quando aplicado em concentrações mais elevadas. Todavia, com os resultados obtidos por Oliveira et al. (2017) com a variedade 'Ubá', observou-se que em temperaturas amenas a porcentagem de florescimento foi incrementada com a aplicação do PBZ.

O estresse hídrico pode ser aliado a aplicação do PBZ na indução floral de mangueiras e consiste na redução gradual da quantidade de água de irrigação aos 80 dias após a aplicação do PBZ, possibilita obter a uniformidade e maturidade dos ramos. A irrigação deve ser reiniciada quando $60 \%$ das gemas apresentarem sintomas de brotação. No entanto, a aplicação do estresse hídrico pode ser restrito a produção em um determinado período do ano porque só pode ser adotado nos meses que não chove Albuquerque e Mouco (2000).

\section{Efeito residual do paclobutrazol no solo.}

Atualmente, estudos reforçam a preocupação ambiental pelo uso de reguladores de crescimento no cultivo da mangueira e a necessidade de analisar os teores residuais do composto antes de uma nova aplicação (Souza et al., 2015). Na realidade, a mobilidade do 
PBZ no solo é pouco conhecido, portanto diagnóstico sobre impactos ambientais resultantes da aplicação de PBZ carecem de estudos.

No estudo realizado por Costa et al. (2008) a quantidade de PBZ lixiviado em solo franco-arenoso foi de $0,83 \%$ do aplicado, essa quantidade sugere que em condições reais de campo a lixiviação é menor do que a prevista quando se considera as características da molécula e sua interação com o solo, como solubilidade, sorção e persistência. No entanto, nesse mesmo estudo o PBZ apresentou potencial de sorção baixo $\left(\mathrm{Kd}=0,83 \mathrm{~L} \mathrm{~kg}^{-1}\right)$, mostrando potencial de mobilidade.

O período de permanência do paclobutrazol no solo depende do tipo de solo e das condições climáticas. Quando o resíduo no solo do paclobutrazol é significativo, observa-se comprometimento do vigor dos brotos. Neste caso, recomenda-se reduzir a dosagem em 50 a $70 \%$ com relação à safra anterior (Albuquerque e Mouco, 2000).

\section{Manejo da poda na indução floral da mangueira}

O manejo da poda pós-colheita é essencial para o sucesso da indução do florescimento da mangueira porque permite obter gemas mais homogêneas e mais férteis, árvores de menor porte para facilitar o manejo da planta, maior arejamento interno e melhor arquitetura da planta. Os ramos produzidos entre 4 a 5 meses após a poda de ponteiros poderão florescer em condições de temperaturas baixas (Davenport, 2006) e ou aplicação do PBZ.

Para obter sucesso no manejo da poda da mangueira, é importante conhecer a morfologia das plantas, como panícula, gema apical, gema axial, primeiro e segundo fluxo. A poda de ponteiros em mangueiras resulta em uma intensa brotação de ramos laterais se os níveis de nitrogênio foliar estiverem entre $1,1 \%$ a 1,4\% e houver disponibilidade de água no solo (Davenport, 2006). Aumento no comprimento e diâmetro dos ramos ocorre durante as duas primeiras semanas e a maturação desses ramos ocorre um a dois meses após a poda (Ramírez \& Davenport, 2010). Além de permitir a renovação da parte aérea da planta, também estimula a renovação das raízes efetivas, as quais são responsáveis pela absorção dos nutrientes e alguns produtos aplicados via solo (Davenport, 2006).

A poda de ponteiros consiste na eliminação de ramos apicais que possuem o diâmetro menor que um centímetro e é usada para sincronizar os surtos de crescimento no dossel da copa, permitindo que todos os ramos no dossel estejam na mesmo fase de desenvolvimento fisiológico em um dado momento (Figura 3). Além de uniformizar o crescimento dos fluxos, 
a poda de ponteiros serve também para remover os ramos pouco vigorosos, doentes e os restos de panículas da floração anterior.

A

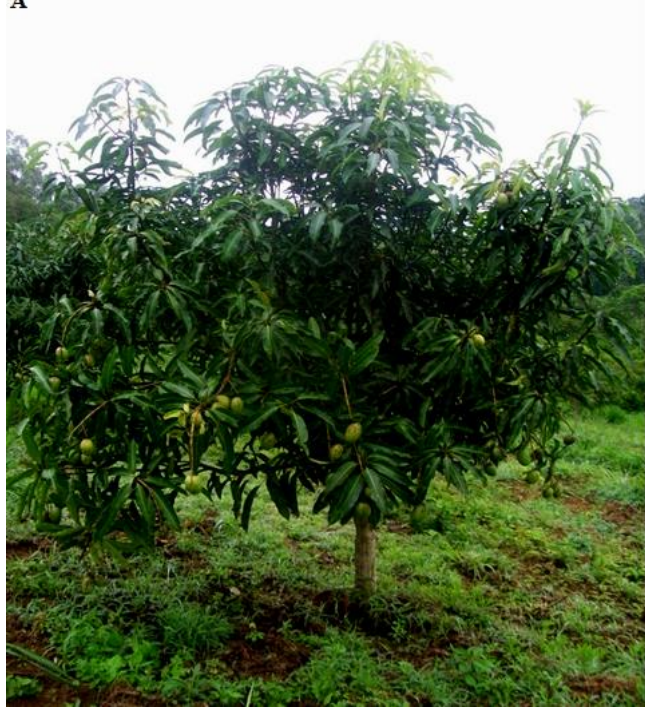

B

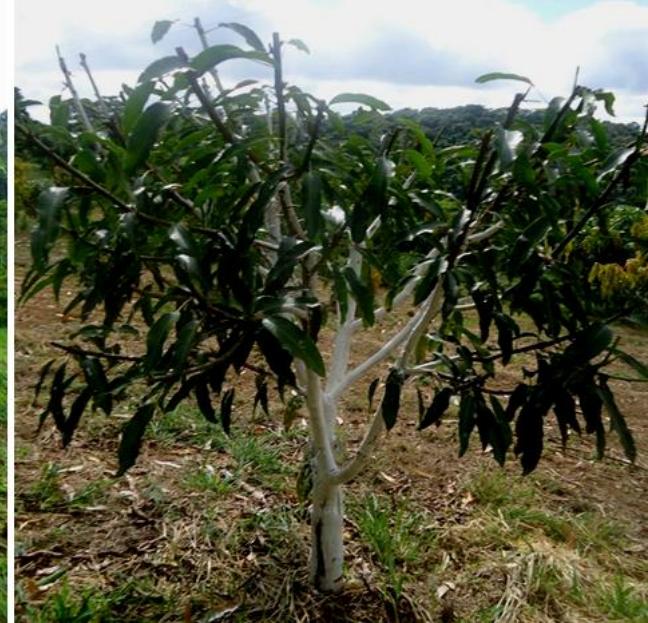

Figura 3. Manejo da poda visando a indução floral de mangueira 'Ubá'; antes (A) e depois (B) da poda. Foto: autoria própria.

O manejo da poda consiste na abertura central da copa em que são retirados os galhos que tenham o ângulo menor do que 45 graus em relação ao tronco para permitir a entrada de luz e o arejamento da copa das árvores, e também, a retirada de ramos doentes e improdutivos. São eliminados os ramos abaixo de 60 centímetros para evitar que as mangas encostem no chão (levantamento da copa). Além disso, deve ser realizado o encurtamento dos ramos laterais e do topo para manter a altura das árvores e o espaçamento de plantio que facilite a condução do pomar nos aspectos fitossanitários e de colheita de frutos.

\section{Considerações Finais}

O paclobutrazol é um produto essencial para o escalonamento e produção o ano todo de manga e é muito conhecido e difundido nas regiões tradicionais de mangicultura no Brasil. A eficiência da utilização do PBZ para induzir o florescimento escalonado e uniforme da mangueira já está bem documentado para as cultivares mais produzidas comercialmente no Brasil. Os fatores inerentes a cultivar, as condições climáticas e até mesmo características do solo são determinantes na recomendação da dose e época de aplicação do produto. Além disso é necessário conhecer a fenologia da cultivar de manga para alcançar a eficiência no manejo 
da indução floral, pois considerando uma mesma cultivar a idade das plantas, o vigor e a nutrição interferem na resposta das plantas a aplicação do PBZ.

Para o sucesso da indução floral da mangueira a aplicação de PBZ deve ser aliado a outros manejos como poda, nutrição mineral e irrigação. Além disso, trabalhos que investiguem o efeito do uso prolongado do paclobutrazol no solo e nas plantas serão importantes para garantir o manejo sustentável da mangicultura brasileira.

\section{Referências}

Anuário Brasileiro de Horti e Fruti 2019. (2019). CEPEA, Piracicaba, São Paulo: Disponível em: http://www.editoragazeta.com.br/produto/anuario-brasileiro-de-horti-fruti-2019/ .

Albuquerque, J.A.S., Mouco, M.A.C. (2000). Manga: indução floral. Petrolina: Embrapa Semi-Árido, 2000. 34p. (Circular Técnica, 47).

Albuquerque, J.A.S., Mouco, M. A. DO C., Medina, V.D., Santos, C.R. Tavares, S.C.C. de H. (1999). O Cultivo da Mangueira Irrigada no Semi-Árido Brasileiro.Embrapa SemiÁrido/VALEXPORT, Petrolina-PE. 77p.

Alburqueque, J. A. S., Medina, V. D., Mouco, M. A. D. (2002). Indução floral. In: Genu, P. J. C.; Pinto, A. C. Q. (Eds.) A cultura da mangueira. Brasília; Embrapa informações tecnológicas, 259-276.

Alburqueque, J. A. S., Mouco, M. A. C. (2005). Efeito do paclobutrazol em duas épocas de produção da mangueira. Revista Bragantia, 64, 219-225.

Asin, L., Alegre, S., Montserrat, R. (2007). Effect of paclobutrazol, prohexadione-Ca, defict irrigation, summer pruning on shoot growth, yield, and return bloom, in a 'Blanquilla' pear orchard. Scientia horticultarae, 113, 142-148.

Cardoso, M. G. S., São José, A. R., VIana, A. E. S., Matsumoto, S. N., Rebouças, T. N. H. (2007). Florescimento e Frutificação de Mangueira (Mangifera indicaL.) cv. Rosa Promovidos por Diferentes Doses de Paclobutrazol. Revista Brasileira de Fruticultura, 29, 209-212. 
Chatzivagiannis, M.A., São José, A.B., Bomfim, M.P., Oliveira Junior, M.X., Rebouças, T.N.H. (2014). Florescimento e produtividade de mangueira 'Boubon', 'Palmer' E 'Rosa' com uso de paclobutrazol. Revista Iberoamericana de Tecnología Postcosecha, 15, 41-47.

Coelho, E.F., Batista, L.S., Alves, A.A.C. (2014). Flowering and fruit set of mango in different doses of paclobutrazol (PBZ). Enciclopédia Biosfera, 10, 1117.

Davenport, T. L. (2007). Reproductive physiology of mango. Brazilian Journal of Plant Physiology, 19, 363-376.

Davenport, T. L. (2006). Pruning Strategies to Maximize Tropical Mango Production from the Time of Planting to Restoration of Old Orchards. Hortscience, 41 (3), 544-548.

Davenport, T. L., Nuñes-Elisea, R. (1997). Reproductive phisiology. In: LITZ, R. E. The Mango. Wallingford: CAB international, 69-121.

De Los Santos-Villalobos, S., Folter, S., Delano-Frier, J., Gómez-Lim, M., Guzmán-Ortiz, D. (2013). Growth Promotion and Flowering Induction in Mango (Mangifera indica L. cv "Ataulfo") Trees by Burkholderia and Rhizobium Inoculation: Morphometric, Biochemical, and Molecular Events. Journal of Plant Growth Regulation, 32, 615- 627.

Fonseca, N., de Castro, M. T. N., Silva C. A. L. (2005). Paclobutrazol e Estresse Hídrico no Florescimento e Produção da Mangueira (Mangifera indica) "Tommy Atkins". Revista Brasileira de Fruticultura, 27, 21-24.

Garcia de Niz, D.A., Esquivel, G.L, Montoya, R.B., Arrieta Ramos, B.G, Santiago, G.A., Gómez Aguilar, J.R., Saão José, A.R. (2014). Vegetative and reproductive development of 'Ataulfo' Mango under pruning and Paclobutrazol Management. Journal of Agricultural Science and Technology, 16, 385-393.

Husen, S., Kuswanto; Ashari, S., Basuki, N. (2012). Induction of Flowering and Yield of Mango Hybrids Using Paclobutrazol. Journal of Agriculture and Food Technology, 2, 153158. 
Mouco, M. A. C., Alburqueque, J. A. S. (2005). Efeito do paclobutrazol em duas épocas de produção da mangueira. Bragantia, 64 (2), 219-225.

Mouco, M. A. C., Ono, E. O. O., Rodrigues, J. D. (2010). Mango flower induction in the brazilian Northeast Semi-arid with gibberellin synthesis inhibitors. Acta horticulture, 884, 591-596.

Mouco, M.A.C., Ono, E.O.O., Rodrigues, J.D. (2011). Controle do crescimento vegetativo e floração de mangueira cv. Kent com reguladores de crescimento vegetal. Revista Brasileira de Fruticultura, 33 (4), 1043- 1047.

Oliveira, G.P., Siqueira, D.L., Salomão, L.C.C., Cecon, P.R., Machado, D.L.M. (2017). Paclobutrazol and branch tip pruning on the flowering induction and quality of mango tree fruits. Pesquisa Agropecuária Tropical, 47 (1), 7-14.

Oliveira, G.P., Siqueira, D.L., Cecon, P.R., Salomão, L.C.C. (2018). Teores de carboidratos em mangueira 'Ubá' submetida a diferentes doses de paclobutrazol. Revista de Ciências Agrárias, 41 (3), 749-756.

Oliveira, M. B., Pereira, M.C.T., Mizobutsi, G.P., Maia. V.M., Silva, J.F., Oliveira, J.A.A., Costa, I.J.S., Nietsche, S., Santos, E.F., Mouco, M.A.C. (2015). Paclobutrazol and tip pruning in the management of 'Palmer' mango trees in the semi-arid region of Brazil. Acta horticulture, 1, 149-156.

Pereira, AS et al. (2018). Metodologia da pesquisa científica. [e-book]. Santa Maria. Ed. UAB/NTE/UFSM. Disponível em:

https://repositorio.ufsm.br/bitstream/handle/1/15824/Lic_Computacao_MetodologiaPesquisa-Cientifica.pdf?sequence $=1$.

Pinto, A. C. Q., Andrade, S. R. M., Amaro, A. A., Gomes, U. (2004). Mango industry in Brazil. Acta Horticulturae, 645, 37-50. 
Pinto, C.A.Q., Silva, D.J., Pinto, P.A.C. (2009). Mangueira. In. Crisostomo, L.A., Naumov, A. (Org) Adubando para alta produtividade e qualidade: Fruteiras tropicais do Brasil . Fortaleza: Embrapa Agroindustrial Tropical, 125-145.

Ramírez, F., Davenport, T. L. (2010). Mango (Mangifera indica L.) Flowering Physiology. Scientia Horticulturae, 126, 65-72.

Ramírez, F., Davenport, T. L., Fischer, G. (2010a). The Number of Leaves Required for Floral Induction and Translocation of the Florigenic Promoter in Mango (Mangiferaindica L.) in a Tropical Climate. ScientiaHorticulturae, 123, 443-453.

Saúco, V.G. El cultivo Del mango. ISBN: 84-7114-841-2. España, 1999. p. 291.

Silva, K.K.A., Ono, E.O., Mouco, M.A.C., Silva, G.J.N., Souza, R.J.M., Silva, N.C., Silva, R.C.B. (2014). Uniconazole no florescimento e produção da mangueira (Mangifera indica L.) cv. Palmer. Revista Megistra, 26 (4), 507-517.

Simões, W.L., de Souza, M.A., Mouco, M.A. do C., de Lima, M.A.C., Calgaro, M. (2017). Paclobutrazol aplicado via sistema de irrigação na indução floral da mangueira 'Keitt'. IV INOVAGRI International Meeting, 2017.

Souza, L.S.S., Silva Neta, C.R., Mouco, M.A., Ferraz, A.V., Silva, P.T.S. (2015).

Concentração de Paclobutrazol no solo de áreas cultivadas com diferentes variedades de mangueira do Vale do São Francisco. X Jornada de Iniciação Científica da Embrapa Semiárido, Petrolina, PE.

Taiz, L., Zeiger, E., Møller, I.A., Murphy, A. (2017). Fisiologia e Desenvolvimento Vegetal. 6 ed. Porto Alegre, 858p.

Whiley, A.W., Schaffer, B. (1997). Stress Physiology, Chapter V. In: Litz, R.E. The Mango, Botany, Production and Uses, CAB International, Wallingford, 147-176.

\section{Porcentagem de contribuição de cada autor no manuscrito}

Girlaine Pereira Oliveira - 100\% 